\title{
Refractory atrial fibrillation following tube thoracostomy
}

\author{
Kuo-Feng Hsu MD, Han-Bin Wang MD, Chung-Bao Hsieh MD
}

Previously published at www.cmaj.ca

A 54-year-old man with a history of cirrhosis following liver transplantation presented with shortness of breath and was found to have a right-sided pleural effusion secondary to pulmonary infection. A pigtail drainage tube was inserted guided by computed tomography. The procedure was complicated by the development of a massive hemopneumothorax for which the patient required an emergency thoracotomy. After two chest tubes (sized 32 French) were inserted, a 12-lead electrocardiogram showed atrial fibrillation and rapid ventricular response (heart rate 150 to 160 beats/min) (Figure 1). The patient had no history of atrial fibrillation.

Laboratory investigations showed serum levels of electrolytes within normal range and oxygen saturation at $97 \%$. Digoxin, amiodarone and verapamil were administered intravenously without any effect on either the heart rate or the rhythm. An anteroposterior chest radiograph showed that the tip of the chest tube was adjacent to the right atrium (Figure $2)$. The chest tube was pulled out, and the cardiac rhythm immediately returned to sinus tachycardia with a rate of 86 beats/min. The atrial fibrillation did not recur.

Arrhythmia following tube thoracostomy is unusual. Three factors in this patient suggested that the refractory arrhythmia was associated with the chest tubes: the short interval between insertion of the tubes and the beginning of the arrhythmia; the proximity of the chest tube to the right atrium; and the immediate termination and no recurrence of the arrhythmia after the tube was withdrawn.

Arrhythmias may result from mechanical stimulation of the pericardium, the heart or the vagus nerve by the chest tube. ${ }^{1}$ The episodes are usually reversible as soon as the stimulus ceases and require no further treatment. ${ }^{2}$ Chest radiographs should be obtained as soon as possible after tube thoracostomy to identify any complications. ${ }^{3}$ If the chest tube is thought to be causing an arrhythmia, it can be pulled back slightly and the effect on the arrhythmia monitored.

From the departments of Surgery (Hsu, Hsieh) and Internal Medicine (Wang), Tri-Service General Hospital, National Defense Medical Center, Taipei, Taiwan, Republic of China

CMAJ 2010. DOI:10.1503/cmaj.090659

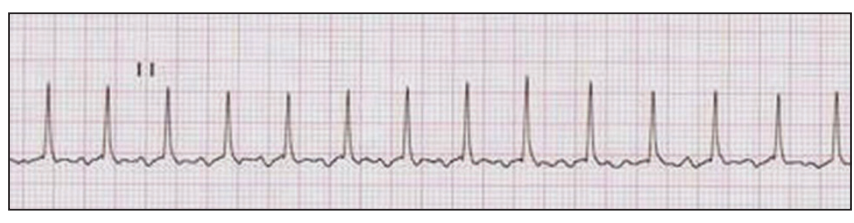

Figure 1: A rhythm strip in a 54-year-old man showing atrial fibrillation with rapid ventricular response of 150 to 160 beats/min in lead II after insertion of chest tubes.

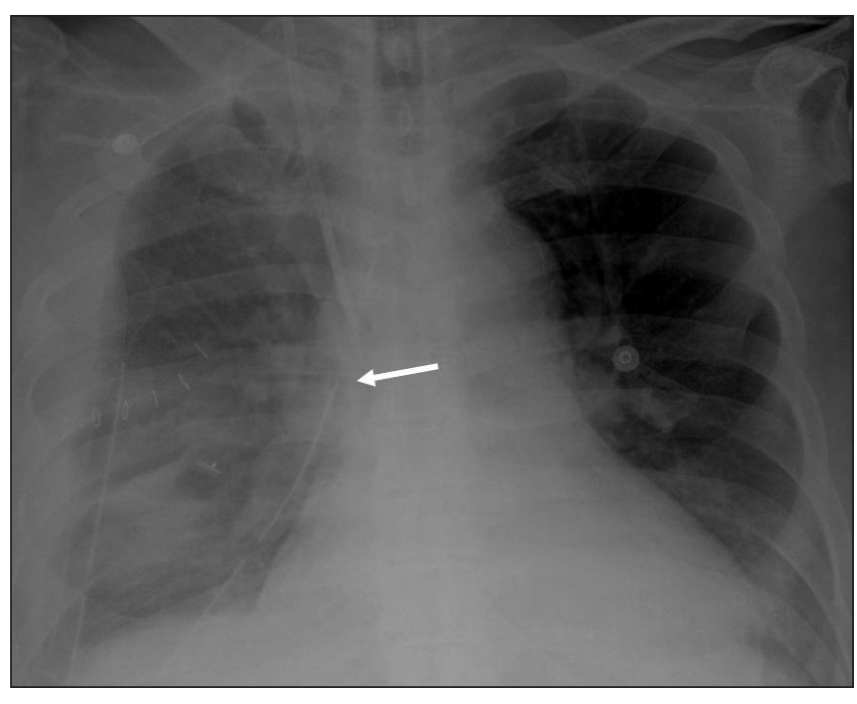

Figure 2: Anteroposterior chest radiograph showing the tip of the chest tube (arrow) adjacent to the right atrium.

This article has been peer reviewed

Competing interests: None declared.

\section{REFERENCES}

1. Ward EW, Hughes TE. Sudden death following chest tube insertion: an unusual case of vagus nerve irritation. J Trauma 1994;36:258-9.

2. Barak M, Iaroshevski D, Ziser A. Rapid atrial fibrillation following tube thoracostomy insertion. Eur J Cardiothorac Surg 2003;24:461-2.

3. Adrales G, Huynh T, Broering B, et al. A thoracostomy tube guideline improves management efficiency in trauma patients. J Trauma 2002;52:210-4. 